\title{
Combination of Western Medicine and Traditional Chinese Medicine for Treatment of Malignant Bone and Soft Tissue Tumors
}

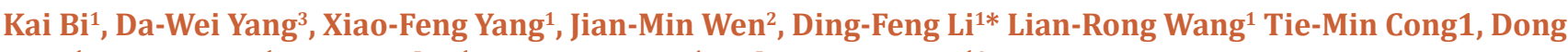 \\ Song1, Yu-Hua Qiu1 ${ }^{1}$, Xu-Bin Chai ${ }^{1}$, Xiao-Hong Liu ${ }^{4}$ and Yan-Jun Zeng'* \\ ${ }^{1}$ Beijing Hospital of Chinese Traditional and Western Medicine, China \\ ${ }^{2}$ Wang Jing Hospital Affiliated to the China Academy of Chinese Medical Sciences, China
}

${ }^{3}$ South Campus of Guang'an men Hospital, China Academy of Chinese Medical Sciences, China

${ }^{4}$ Parkway Health Hongqiao Medical Center, China

Submission: January 25, 2018; Published: February 16, 2018

*Corresponding author: Ding-feng Li, Beijing Hospital of Chinese Traditional and Western Medicine, 3 Yong Ding Rd, Beijing, China, Email: 307yygk@sina.com

Yan-jun Zeng, Beijing Hospital of Chinese Traditional and Western Medicine, 3 Yong Ding Rd, Beijing, China, Email: yjzeng@bjut.edu.cn

Abstract

Aim: To discuss the effect of the combination of neoadjuvant intra-arterial chemotherapy by subcutaneous implantable delivery system (SIDS) and Traditional Chinese Medicine (TCM) in treatment of malignant bone and soft tissue tumors.

Method: from August 2001 to February 2016, applying neoadjuvant intra-arterial chemotherapy by SIDS combined with Traditional Chinese Medicine to 148 patients with malignant bone and soft tissue tumors.

Results: based on clinical symptoms, videos and laboratory tests data collected after patients have received the combined treatment, in reference to the Response Evaluation Criteria in Solid Tumors (RECIST) of the National Cancer Institute (NCI), we observed 9 patients went into complete response (CR), 120 patients went into partial response (PR), RR reached $87.2 \%$ and DCR reached $100 \%$. We observed side effects, including Myelosuppression. Leucopenia of grade 1 or 2 occurred on 46 patients (31.08\%), leucopenia of grade 3 or 4 occurred on 15 patients $(10.13 \%)$, thrombocytopenia of grade 1 or 2 occurred on 22 patients $(14.08 \%)$, and thrombocytopenia of grade 3 or 4 occurred on 3 patients (2.03\%). Hair loss occurred on all 148 patients (100\%). Myalgias or arthralgias occurred on 24 patients (16.22\%). Liver injury of grade 1 or 2 occurred on 6 patients (4.05\%). Gastrointestinal reactions of grade 1 or 2 occurred on 16 patients $(10.81 \%)$.

Conclusion: In treatment of malignant bone and soft tissue tumors, intra-arterial chemotherapy by SIDS combined with TCM can alleviate side effects or untoward effects like gastrointestinal reactions, myelosuppression, reduce damages to heart, liver, kidney and urinary system, and strengthen immune system, creating a synergistic effect.

Keywords: Intra-arterial chemotherapy by subcutaneous implantable delivery system; Malignant bone tumors; Malignant soft tissue tumors

Abbreviations: SIDS: Subcutaneous Implantable Delivery System; TCM: Traditional Chinese Medicine; VAS: Visual Analogue Scale; RECIST: Response Evaluation Criteria in Solid Tumors; NCI: National Cancer Institute; CR: Complete Response; PR: Partial Response

\section{Introduction}

With an estimated incidence of four to five cases per million people per year, osteosarcoma is the most common primary bone tumor [1]. The incidence of soft tissue tumors is low, accounting for $1 \%$ of adult malignancies and $15 \%$ of pediatric malignancies [2]. Mortality, however, is high; the average fiveyear survival rate is only $60 \%$ [3]. Before the introduction of (neo-) adjuvant chemotherapy in the late 1970s, the long-term cure rate of non-metastatic osteosarcoma was $10-25 \%$ [4]. In recent years, advantages of neoadjuvant chemotherapy, which are suggested by Rosen, has been questioned and challenged by some scholars [5,6]. Neoadjuvant chemotherapy, having been applied in treatment of malignant bone and soft tissue tumors, have greatly increased the survival rate of patients, but also caused severe side effects, even obstinate complications. From August 2001 to February 2016, when neoadjuvant chemotherapy 
by SIDS was applied on 148 patients with malignant bone and soft tissue tumors, traditional Chinese medicine was also provided before and after the chemotherapy process to strengthen body resistance and prevent the attack of pathogenic factors, reinforce vital energy and enrich blood and tonify liver and kidney. The clinical effect of the combined treatment was relatively satisfactory.

\section{Patients and Methods}

\section{Patients}

From August 2001 to February 2016, 148 patients with malignant bone and soft tissue tumors have been received and treated. Among the 148 patients, 82 male, 66 female, age ranging from 6 to 76,55 patients were with osteosarcoma, 32 with chondrosarcoma, 21 with Liposarcoma, 13 with Ewing's sarcoma, 7 with malignant fibrous histiocytoma, 6 with rhabdomyosarcoma, 6 with PNET, 5 with malignant lymphoma, 3 with synovial sarcoma. The length of follow-up medical care for patients ranged from 17 months to 6 years with 4 months, 28 months on average.

\section{Methods}

The Minimum Standards of Reporting Checklist contains details of the experimental design, and statistics, and resources used in this study (Additional file 1). Clinical Diagnoses- The diagnoses of malignant bone and soft tissue tumors were confirmed with the help of imaging examinations, including $\mathrm{x}$-ray, CT scan and MRI scan and clinical evaluations. Arterial Intubation Operation with Subcutaneous Implantation of Chemotherapy Pump Neoadjuvant chemotherapy by SIDS- an intra-arterial infusion pump was implanted subcutaneously based on the location of tumors. For all patients, retrograde catheterization via the femoral or brachial artery was adopted. The catheter was connected with the chemotherapy pump, and the pump was implanted beneath the skin around the incision.

\section{Chemotherapeutic Protocol}

The induction chemotherapy cycle includes pirarubicin or epirubicin $[50-70 \mathrm{mg} / \mathrm{m} 2$ by 4 -hour intra-arterial infusion/day, for 3 days] and nedaplatin [100-120 mg/m2 by 2-hour intraarterial infusion/day, for 3 days]. The cycle has been repeated every 2-3 weeks. Specifics were determined according to the nature of tumors and immunohistochemical results. Different medicines to protect myocardium, liver, kidney and urinary system and to stop vomiting were provided according to different chemotherapeutic agents. 3 cycles made up a treatment course. Basically, 1-2 courses were needed both before and after the surgery.

\section{Traditional Chinese Medicine}

TCM was used before the intra-arterial chemotherapy by SIDS. Decoct the following herbs: radix astragali $30 \mathrm{~g}$, radix pseudostellariae $30 \mathrm{~g}$, radix codonopsis pilosulae $15 \mathrm{~g}$, radix glehniae $15 \mathrm{~g}$, radix ophiopogonis $10 \mathrm{~g}$, radix polygonati officinalis
$10 \mathrm{~g}$, fruits of Chinese privet $10 \mathrm{~g}$, fruits of Chinese wolfberry $10 \mathrm{~g}$, prepared tuber of multiflower knotweed $10 \mathrm{~g}$, and ecliptae herba $10 \mathrm{~g}$. One dose every day, each dose divided into 3 portions, every portion taken after meals. The medicine recipe can be adjusted according to reactions of each individual patient after they have received the chemotherapy. For patients who vomit, we can add radix aucklandiae by $8 \mathrm{~g}$ and bamboo shavings by $10 \mathrm{~g}$; for patients with severe vomiting, we can add inula flower by $10 \mathrm{~g}$, persimmon calyx by $3 \mathrm{~g}$, bamboo shavings by $10 \mathrm{~g}$, and rhizoma pinelliae preparata by $8 \mathrm{~g}$; for patients with poor appetite, we can add rose by $10 \mathrm{~g}$, neroli by $10 \mathrm{~g}$ and finger citron flower by $10 \mathrm{~g}$; for patients with abdominal distension who cannot take any food, we can add charred triplet by $10 \mathrm{~g}$, rhizoma cyperi by $6 \mathrm{~g}$, perilla stem by $10 \mathrm{~g}$ and dried orange peel by $6 \mathrm{~g}$; for patients with generally poor situation but no specific adverse reactions, we can add red ginseng by $15 \mathrm{~g}$ and American ginseng by $10 \mathrm{~g}$; for patients with generally good situation, well appetite and no vomiting, we can add polygonum cuspidatum by $15 \mathrm{~g}$, black nightshade by $10 \mathrm{~g}$, paris polyphylla smith by $15 \mathrm{~g}$, Chinese lobelia by $10 \mathrm{~g}$, scutellariae barbatae by $10 \mathrm{~g}$ and hedyotis diffusa by $10 \mathrm{~g}$.

Results

\section{Standards for Effect Evaluation}

i. To evaluate the treatment effects, X-ray imaging, CT and MRI were used to measure the size of tumors, and the appraised effects of middle stage have also been taken into consideration. In reference to the RECIST of NCI, treatment results fell into 4 categories as complete response (CR), partial response (PR), stable disease (SD) and progressive disease (PD). Under CR, all target lesions have disappeared; Under PR, based on images and videos, at least a $30 \%$ decrease in the sum of the longest diameter of target lesions has been observed, or lymph nodes or distant metastases have disappeared; under PD, based on images and videos, a $20 \%$ or greater increase in the sum of the longest diameter of target lesions has been observed, or one or more lesions have appeared; under SD, based on images and videos, neither sufficient shrinkage to qualify for PR or sufficient increase to qualify for PD has been observed. Based on CR and $P R$, response rate (RR) can be figured out, and based on CR, PR and SD, disease control rate (DCR) can be figured out.

ii. Visual Analogue Scale (VAS) is applied to measure the pain intensities of patients before and after the chemotherapy.

iii. Impacts on body systems and side effects caused by chemotherapy have been recorded.

\section{Treatment Results}

\section{Effects}

In reference to the RECIST of NCI, among the 148 patients, 9 patients went into CR (Figure 1), 120 patients went into PR (Figure 2), and 19 patients went into SD. RR reached $87.2 \%$, 


\section{Orthopedics and Rheumatology Open Access Journal}

and DCR reached $100 \%$. The length of follow-up medical care for patients ranged from 17 months to 6 years with 4 months, 28 months on average. After surgeries, 25 patients suffered metastasis, relapse or death, while on others no relapse or metastasis was observed.

\section{VAS for Pain Measurement}

VAS scores before chemotherapy ranged from 4 to 10 points, $7.8 \pm 2.6$ points on average. VAS scores after chemotherapy ranged from 0 to 5 points, $2.6 \pm 1.4$ points on average, with statistical significance $(\mathrm{P}<0.05)$ (Figure 1$)$
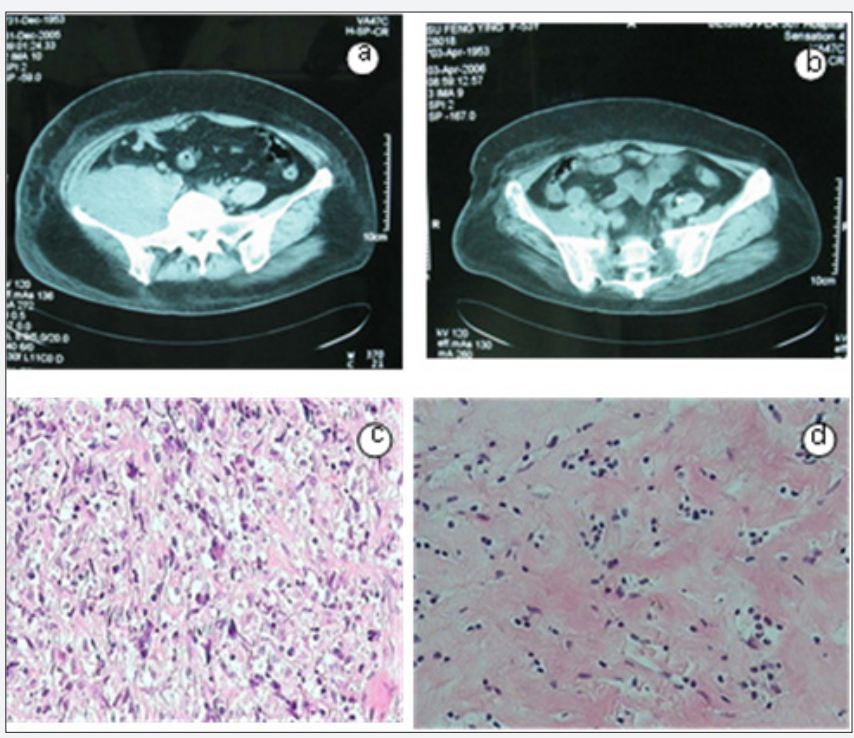

Figure 1: Liposarcoma case.

a. CT image of a female with a large myxiod liposarcoma in pelvis taken before the patient received the chemotherapy by SIDS and TCM.

b. CT image taken after 3 times of the chemotherapy by SIDS and TCM shows the space occupied by the tumour has shrink and boundary of the tumor is clear.

c. Image shows abnormal cell proliferation before the chemotherapy by SIDS and TCM.

d. Image shows tissue necrosis and fibrosis after the chemotherapy by SIDS and TCM.

Figure 2 : Osteosarcoma case.

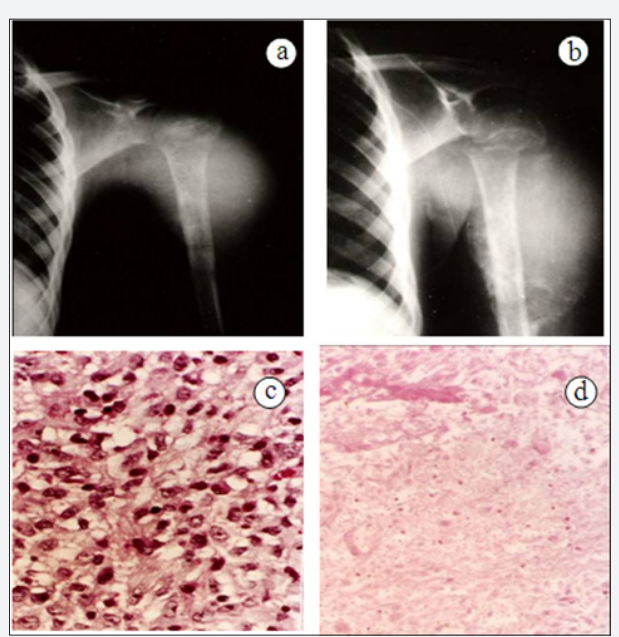

a. X-ray image of a male with osteosarcoma in proximal humerus taken before the patient received the chemotherapy by SIDS and TCM

b. X-ray image taken after 6 times of the chemotherapy by SIDS and TCM shows the space occupied by the tumor has shrink, pathological fracture is visible and boundary of the tumor is clear.

c. Image shows abnormal cell proliferation before the chemotherapy by SIDS and TCM.

d. Image shows tissue necrosis and fibrosis after the chemotherapy by SIDS and TCM. 


\section{Side Effects}

The chemotherapy may cause life-threatening reactions, potential reactions and adverse reactions that are quite severe. The fatal complications caused by the chemotherapy are mainly infection and hemorrhage which didn't occur in this treatment process. Side effects caused by the neoadjuvant chemotherapy by SIDS include:

i. Myelosuppression. Leucopenia of grade 1 or 2 occurred on 46 patients (31.08\%), leucopenia of grade 3 or 4 occurred on 15 patients (10.13\%), thrombocytopenia of grade 1 or 2 occurred on 22 patients (14.08\%), and thrombocytopenia of grade 3 or 4 occurred on 3 patients $(2.03 \%)$.

ii. Hair loss occurred on all 148 patients (100\%).

iii. Myalgias or arthralgias occurred on 24 patients (16.22\%).

iv. Liver injury of grade 1 or 2 occurred on 6 patients (4.05\%).

v. Gastrointestinal reactions of grade 1 or 2 occurred on 16 patients $(10.81 \%)$.

\section{Discussion}

Chemotherapy can extend the survival time of bone tumor patients, and pre-operative chemotherapy could further facilitate the application of limb salvage treatment. So far, 85$90 \%$ patients with malignant tumors are suitable for limb salvage treatment through neoadjuvant chemotherapy [7]. Due to the advantages of intra-arterial infusion chemotherapy (including high local concentration, powerful anticancer effect, evitable liver degradation, less toxic and side effects than infusion via vein, shortened interval between chemotherapy and continuous drug delivery) [8], compared with intravenous administration, intra-arterial administration can achieve high-concentration of chemotherapeutic agents and contribute to tumor penetration directly by physical diffusion, and promote tumor cell destruction on molecular basis, without drug inactivation and degradation in liver [9]. We employed it and TCM as a preoperative procedure.

Though SIDS has not been popular yet, neoadjuvant chemotherapy has already been put into broad practice. Unfortunately, in recent decades the long-time event-free survival of osteosarcoma patients has reached a plateau at $60-70 \%$ [10]. The clinical effect of the chemotherapy is far from expectations, as different levels of drug resistance have occurred on patients and side effects caused by the chemotherapy have significantly affected the life quality and survival rate of patients. Thus, the endeavor to salvage poor responders by postoperative therapy adaptation belongs to the major challenges in osteosarcoma treatment today and finds its expression in current therapy-optimisation trials [11]. In this regard, traditional Chinese medicine plays an important role as TCM can effectively enhance anti-tumor effects, alleviate adverse reactions caused by chemotherapy, and strengthen the body's functions by tonifying internal organs like spleen, stomach, liver and kidney to replenish vital energy and enrich blood in the body. TCM can help overcome the limitations and drawbacks of the chemotherapy.

\section{TCM Helps Alleviate Side Effects}

In our study, 148 paints with malignant bone and soft tissue tumors have received the combined treatment of the neoadjuvant chemotherapy by SIDS and traditional Chinese medicine. Among them, gastrointestinal reactions of grade 1 or 2 occurred on 16 patients, $10.81 \%$ of all patients. During the chemotherapy process, the Chinese medicine recipe was adjusted according to different reactions of patients to alleviate nausea, vomiting, palpitation, headache, fatigue and other side effects to make sure chemotherapy can proceed smoothly. For patients who vomit, we can add radix aucklandiae by $8 \mathrm{~g}$ and bamboo shavings by $10 \mathrm{~g}$; for patients with severe vomiting, we can add inula flower by $10 \mathrm{~g}$, persimmon calyx by $3 \mathrm{~g}$, bamboo shavings by $10 \mathrm{~g}$, and rhizoma pinelliae preparata by $8 \mathrm{~g}$; for patients with poor appetite, we can add rose by $10 \mathrm{~g}$, neroli by $10 \mathrm{~g}$ and finger citron flower by $10 \mathrm{~g}$; for patients with abdominal distension who cannot take any food, we can add charred triplet by $10 \mathrm{~g}$, rhizoma cyperi by $6 \mathrm{~g}$, perilla stem by $10 \mathrm{~g}$ and dried orange peel by $6 \mathrm{~g}$. The final results affirmed that TCM can alleviate side effects like vomiting and poor appetite caused by chemotherapy.

In our study, Leucopenia of grade 1 or 2 occurred on 46 patients $(31.08 \%)$, leucopenia of grade 3 or 4 occurred on 15 patients $(10.13 \%)$, thrombocytopenia of grade 1 or 2 occurred on 22 patients $(14.08 \%)$, thrombocytopenia of grade 3 or 4 occurred on 3 patients $(2.03 \%)$, and liver injury of grade 1 or 2 occurred on 6 patients (4.05\%). TCM is effective in treating hepatic dysfunction and leucopenia which are caused by chemotherapy, especially in increasing the number of white blood cells and reducing transaminase. Fruits of Chinese privet, fruits of Chinese wolfberry, prepared tuber of multiflower knotweed and ecliptae herba can help replenish blood and generate marrow, and thus are specifically chosen for treatment. From the reactions of patients, we can conclude that TCM can to some extent ease myelosuppression and some severe impacts on heart, liver, kidney and urinary system caused by the chemotherapy.

In our study, among 148 patients, 9 patients went into CR, 120 went into $\mathrm{PR}$, and 19 went into SD, and RR reached $87.2 \%$, and DCR reached $100 \%$. The neoadjuvant chemotherapy produced effects, and TCM can strengthen the anti-tumor effects. For patients with generally poor situation but no specific adverse reactions, we can add red ginseng by $15 \mathrm{~g}$ and American ginseng by $10 \mathrm{~g}$; for patients with generally good situation, well appetite and no vomiting, we can add polygonum cuspidatum by $15 \mathrm{~g}$, black nightshade by $10 \mathrm{~g}$, paris polyphylla smith by $15 \mathrm{~g}$, Chinese lobelia by $10 \mathrm{~g}$, scutellariae barbatae $10 \mathrm{~g}$ and hedyotis diffusa by $10 \mathrm{~g}$. TCM can inhibit the growth of cancer cells and kill them. We didn't organize a systematic clinical test to prove 
the effects of TCM, as we thought even the effects were affirmed by the test, it still seems subjective. However, the effects of TCM should not be underestimated, especially in solving drug resistance in chemotherapy. TCM can strengthen the anti-tumor effect of chemotherapy and alleviate allergic reactions.

Among 148 patients, several were allergic to nedaplatin, and for a few others, their bodies were resistant to chemotherapeutic agents. After TCM was applied, allergic reactions disappeared and the size of tumor has shrink, which proved the reliability of TCM. The combined treatment of the chemotherapy and TCM can kill cancer cells to the maximum limit, boost the immune system, protect patients against infections, reduce malignant tumor exudation, minimize side effects caused by the chemotherapy and improve the internal environment of the body to eliminate tumors or slower their growth, improve life quality, extend lifespan and increase survival rate.

\section{TCM Helps Regulate Patients' Immune System}

Vital energy is important to cellular immune functions of the patients with malignant bone and soft tissue tumors, and the immune functions of cells will impact the occurrence, development and prognosis of tumors. Lowered immunity impacts the treatment of malignant tumors, too. TCM not only has an effect on immune cells, but can also coordinates immune functions of the whole body system through multiple targets like cytokine to help patients enhance vital energy and immunity, and thus TCM is getting growing attention. Many scholars have already done a lot of clinical and experimental research about TCM's immunomodulatory effects in treatment of malignant tumors. TCM mainly works on cellular immunity, basically nonspecific immunity. Compared with immunopotentiator, TCM's effect is relatively weak.

\section{Synergistic Effect}

Based on research, many scholars have found some Chinese herbs or components through external application can help chemotherapeutic agents to inhibit the growth of cancer cells, and if taken orally, they can help experimental animals improve the sensibility to chemotherapeutic agents and alleviate tumors. Both experimental researches and clinical observations have proven the remarkable complementarities of TCM and chemotherapy. TCM complements chemotherapy in a multi-target, multi-channel and multi-link manner to create a synergistic effect.

\section{Conclusion}

In summary, the effect of the combined treatment of the neoadjuvant chemotherapy by SIDS and TCM for malignant bone and soft tissue tumors has been proven positive. TCM works in a multi-target, multi-channel and multi-link manner to improve cellular immunity, induce the production of interferon, increase the production of cytokines, strengthen humoral immunity, inhibit the growth of tumors, promote wound repair, fight against infections, reduce exudation of malignant tumors and improve appetite, so TCM is conductive to patients' general rehabilitation. We believe that Chinese herbs can strengthen body resistance and prevent the attack of pathogenic factors, reinforce vital energy and enrich blood and tonify liver and kidney, and they can help chemotherapeutic agents to kill cancer cells, reduce side effects and enhance body immunity, creating a synergistic effect with chemotherapy. To further increase the survival rate and life quality for patients after they received the chemotherapy by SIDS is a development trend in the treatment of malignant bone and soft tissue tumors.

\section{Authors' Contributions}

Kai Bi provided to experimental ideas and design partially, performed the experiments for TCM helps prescription, wrote the manuscript. Ding-feng Li performed SIDS system and formulate chemotherapeutic protocol for patients, also clinical diagnoses. Dong Song, Yu-hua Qiu and Xu-bin Chai management of patients with chemotherapy and traditional Chinese Medicine. Da-wei Yang, Xiao-feng Yang, and Xiao-hong Liu analyzed the data. Lian-rong Wang and Tie-min Cong analyzed synergistic effect. Jian-min Wen provided many ideas and discussion. Yanjun Zeng contributed to supervision, discussion and reviewed/ edited the manuscript. Both authors read and approved the final manuscript.

\section{Acknowledgement}

We would like to express our acknowledgements to Prof. Wei-dong for the analysis and interpretation of data.

\section{Competing Interests}

The authors declare that they have no competing interests.

\section{Availability of Data and Materials}

Please contact author for data requests.

\section{Consent for Publication}

Not applicable.

\section{Conflict of Interest statement}

There is no conflict of interest among the authors of this study and no objection to the selection and order of the authors.

\section{Funding}

This research was supported by The Fund for Beijing Science \& Technology Development of TCM (JJ2016-21).

\section{Ethics Statement}

Written consent was obtained from the family members of all patients, and the study was approved by the Medical Ethics Committee of the Beijing Hospital of Chinese Traditional and Western Medicine.

\section{References}

1. Bacci G, Ferrari S, Longhi A, Forni C, Ruggieri P, et al. (2003) Preoperative therapy versus immediate surgery in nonmetastatic osteosarcoma. J Clin Oncol 21(24): 4662-4663. 
2. Benjamin R, Pisters P, Helman LJ (2008) Sarcomas of Soft Tissue. Clinical Oncology, p. 4-56.

3. Carrle D, Bielack SS (2006) Current strategies of chemotherapy in osteosarcoma. Int Orthop 30(6): 445-451.

4. Cui Q, Li D, Zhang J, Wang X, Liu S, et al. (2012) The Significance of Preoperative Chemotherapy in Evaluation of Recurrent Soft Tissue Liposarcoma Necrosis. Pathol Oncol Res 18(3): 629-633.

5. David D, Rajappan LM, Balachandran K, Thulaseedharan JV, Nair AS, et al. (2011) Prognostic significance of STAT3 and phosphorylated STAT3 in human soft tissue tumors-a clinicopathological analysis. J Exp Clin Cancer Res 30: 56.

6. Fletcher C, Unni K, Mertens F (2002) World Health Organization classification of tumours. Pathology and genetics of tumours of soft tissue and bone. IARC Press, Lyon, France, pp. 264-284.

7. Goorin AM, Schwartzentruber DJ, Devidas M, Gebhardt MC, Ayala AG, et al. (2003) Presurgical chemotherapy compared with immediate surgery and adjuvant chemotherapy for nonmetastatic osteosarcoma: Pediatric Oncology Group Study POG-8651. J Clin Oncol 21: 1574-1580.

8. Laux CJ, Berzaczy G, Weber M, Lang S, Dominkus M, et al. (2015) Tumour response of osteosarcoma to neoadjuvant chemotherapy evaluated by magnetic resonance imaging as prognostic factor for outcome. Int Orthop 39(10): 97-104.

9. Li D, Cui Q, Liu Y, Wang X, Liu C, et al. (2011) Chemotherapy Response Analysis for Osteosarcom with Intra-arterial Chemotherapy by Subcutaneous Implantable Delivery System December. Pathol Oncol Res 17(4): 947-953.

10. Liu C, Cui Q Shu C, Guo J, Li D, et al. (2013) Comprehensive Treatment Based on Intra-arterial Chemotherapy for Distal Femur Neoplasms. Pathol Oncol Res 19(3): 489-493.

11. Marina N, Bielack S, Whelan J, Smeland S, Krailo M, et al. (2010) International collaboration is feasible in trials for rare conditions: the EURAMOS experience. Cancer Treat Res 152: 339-353.

\section{Your next submission with Juniper Publishers will reach you the below assets}

- Quality Editorial service

- Swift Peer Review

- Reprints availability

- E-prints Service

- Manuscript Podcast for convenient understanding

- Global attainment for your research

- Manuscript accessibility in different formats

( Pdf, E-pub, Full Text, Audio)

- Unceasing customer service

Track the below URL for one-step submission https://juniperpublishers.com/online-submission.php 